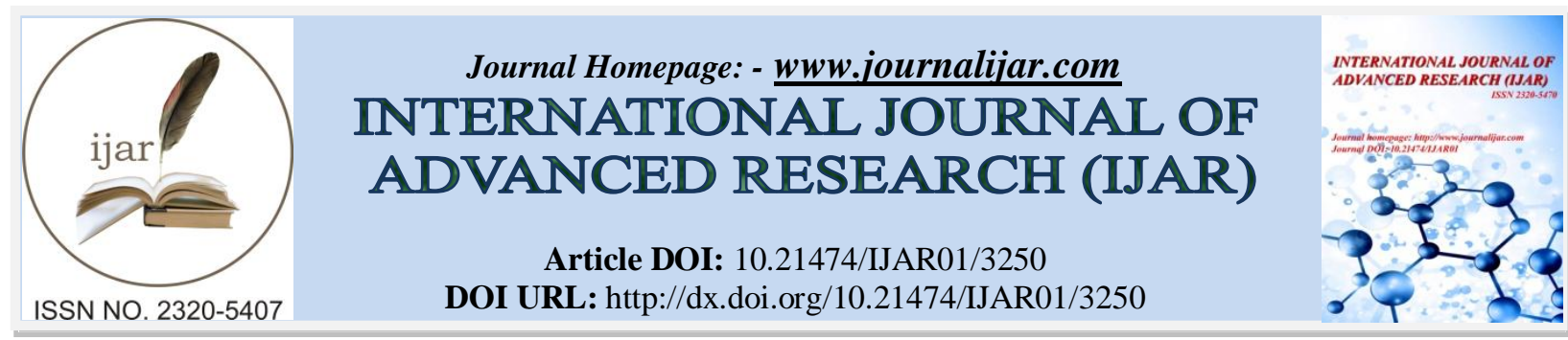

RESEARCH ARTICLE

\title{
HOW ODONTOBLASTS REACT ULTRA-STRUCTURALLY TO ORTHODONTIC INTRUSIVE FORCES.
}

Tarek H. Marei ${ }^{1}$, Heba A. Adawy ${ }^{2}$ and Dina M. Abdel khalik ${ }^{3}$.

1. Associate Professor, Department of Orthodontics, Faculty of Dental Medicine for Girls, Al-Azhar University, Cairo, Egypt.

2. Associate Professor, Department of Oral and Dental Biology, Faculty of Dental Medicine for Girls, Al-Azhar University, Cairo, Egypt.

3. Lecturer, Department of Oral and Dental Biology, Faculty of Dentistry, Ain Shams University, Cairo, Egypt.

\section{Manuscript Info}

Manuscript History

Received: 12 December 2016

Final Accepted: 23 January 2017

Published: February 2017

Key words:-

Odontoblasts, TEM, tooth intrusion, orthodontic force.

\section{Abstract}

Background: The purpose of orthodontic treatment is to move teeth as efficiently as possible with least damage to teeth and their supporting tissues. Previous studies have revealed that varying degrees of force magnitude, frequency, and duration of orthodontic forces express extensive macroscopic and microscopic changes on dental tissues.

Aim of the work:The present work aimed to investigate the ultrastructural changes that might occur to odontoblasts in response to light and heavy intrusive orthodontic forces.

Materials and Methods: Twenty-four vital maxillary first premolars from orthodontic extraction cases were allocated in three groups. Control group: premolars were not subjected to any force. Group I: light intrusive force was applied. Group II: heavy intrusive force was applied. Extraction was done after three weeks of force application. Coronal and radicular pulpal tissues were examined by transmission electron microscope (TEM).

Results: Control, TEM revealed common cellular features regarding coronal and radicular odontoblasts. Evidence of affection of the odontoblasts could be manifested in both experimental groups as they expressed varies degrees of nuclear alterations, chromatin condensation, cytoplasmic organelles affection and loss of cellular junctions.

Conclusions: Intrusive orthodontic force can induce ultra-structural changes in odontoblastic cells. Heavy intrusive have greater impact on odontoblastic cells than light force. Generally, radicular cells are more affected than coronal cells by the same magnitude of the intrusive force. Both coronal and radicular odontoblastic cells can transform into multinucleated odontoclastic cells under the influence of heavy intrusive orthodontic force. Light intrusive force could induce odontoclastic differentiation of radicular odontoblastic cells.

Copy Right, IJAR, 2017,. All rights reserved. 


\section{Introduction:-}

The purpose of orthodontic treatment is to move teeth as efficiently as possible with least damage to teeth and their supporting tissues. Long ago, Orban and Bhaskar,1976 stated that orthodontic forces had no effect on dental pulp but since then, several studies have evaluated the impact of orthodontic forces on dental tissues. These tissues, when exposed to varying degrees of orthodontic force magnitude, frequency and duration of mechanical loading, express extensive macroscopic and microscopic changes (Krishnan et al., 2006). Then, it was confirmed that the magnitude of applied force does not need to be excessive; even small forces of short duration of around 4 hours may be adequate to evoke cellular responses (Gajapuradal et al., 2016).

Orthodontic forces are capable of stimulating the whole vascular system in the dental pulp, in the form of circulatory vascular stasis to necrosis (Andreasen et al., 2007). It was found that there is an increase in expression of sensory neuropeptides, such as Substance P (SP) and calcitonin gene-related peptide, in dental pulp following orthodontic force application in rats (Yamaguchi and Kasai ,2007).

A wide variety of morphological changes have been documented histologically, in response to orthodontic force, affecting both the inner and outer layers of the pulpal tissue. The majority of these changes are deleterious in nature. The odontoblast expressed varying degrees of vacuolization, degeneration, distortion, separation and elongation (Stenvick et al., 1970) ,(Mostafa et al., 1991), (Shigehara et al., 2006) and (Ramazanzadeh et al., 2009). These alterations have been more pronounced in the coronal pulp tissue (Villa et al.,2005). It is proposed to be compensated by osteopontin release which stimulates differentiation of new odontoblasts, thereby helping repair of any tissue damage (Shigehara et al., 2006). These deleterious pulpal changes were more noticeable in older patients, possibly due to a reduction in the size of the apical foramen, with concurrent constriction of apical vessels. Therefore, fortunately, younger patients, who are more susceptible to orthodontic treatment, could be at less risk (Hamilton et al., 1999).

Although tooth intrusion is a special and critical type of orthodontic tooth movement, it was thought in the past that intrusion is clinically impossible to occur( Proffit et al., 2012). Now, Intrusion is thought to have the greatest impact on internal root resorption (Harris et al., 2006). Internal root resorption is the progressive destruction of radicular dentin along the middle and apical thirds of the canal walls as a result of odontoclastic cells activities or due to "strangulation" of the pulp by occluding the apical blood supply (Veberiene et al., 2009). From a histological perspective, internal root resorption is manifested in one form that is purely destructive, internal (root canal) inflammatory resorption, and in another, that is accompanied by repair, internal (root canal) replacement resorption that is featured by the deposition of metaplastic bone/cementum-like tissues adjacent to the sites of resorption (Patel et al., 2010).

Ramazanzaeh et al.,2009 reported no statistically significant difference between the effect of extrusive and intrusive orthodontic tooth movements on the dental pulp except for the presence of more fibrosis in the extrusive force cases. Recent histological study (Lazzaretti et al.,2014) concluded that orthodontic intrusion force caused vascular changes in the pulpal tissue and also increased the presence of fibrosis and the number of pulp calcifications after 21 days.

According to the done database search, there is relatively little data in the literature regarding the odontoblastic response, especially ultra-structurally, to different magnitudes of intrusive orthodontic tooth movement.

\section{Aim of the work:-}

The present work aims to investigate the ultra-structural changes that might occur to odontoblasts in response to light and heavy intrusive orthodontic forces. This could help orthodontists to evaluate pulp response to orthodontic forces of different magnitudes, and accordingly case prognosis.

\section{Materials and Methods:-}

Twenty-four vital and sound maxillary first premolars, from orthodontic male patients (15-22 years old) treated in the Department of Orthodontics, Faculty of Dental Medicine for Girls, Al-Azhar University, Cairo, Egypt, were included in the study. All of the included premolars were selected to be minimally malposed, out of any occlusal trauma and were planned for extraction during comprehensive orthodontic treatment. All cases were selected to be Angle's class I dento-alveolar bimaxillary protrusion with minimal crowding. All patients had good oral hygiene, no previous orthodontic treatment, no history of dental trauma, no missing permanent teeth (with exclusion of $3^{\text {rd }}$ 
molars), no serious inflammatory systemic or oral conditions. The patients were instructed to stop taking any antiinflammatory drugs 1 month before bonding till extraction.

The study proposal was approved by the Research Ethics Committee (REC), Faculty of Dental Medicine for Girls, Al-Azhar University, Cairo, Egypt. An informed consent was signed by the patient or his guardian after treatment and research procedures were explained to him/her.

\section{Grouping:-}

Only one premolar from each patient was included in the study and randomly allocated in one of three groups $(\mathrm{n}=8$ for each). First is the control group, in which the maxillary first premolars were not bonded and extracted just prior to the leveling and alignment stage. Second, Group I, in which light intrusive force was applied to the test premolars. Third, Group II, in which heavy intrusive force was applied to the test premolars.

\section{Appliances and loading:-}

All patients were treated with synergy low friction straight wire (LFSW) appliances with 0.022" slot (Rocky Mountain Orthodontics RMO Corp., Colorado, USA ).

In Group I, the test premolars were not bonded until the 0.012" NiTi initial archwire (Ortho Technology, Inc., Florida, USA) was fully engaged in all other bracket slots. Then, the bracket of the test premolar was bonded in a position which allows the bracket slot to be $1 \mathrm{~mm}$ occlusal to the archwire level and parallel to it. At the same session, the archwire was forcefully engaged in the slot of the newly bonded bracket giving light intrusive force as measured clinically. Measuring the force was done before bonding of the test premolar by deflecting the archwire segment between canine and second premolar brackets $1 \mathrm{~mm}$ occlusally using a wire tucker. Then a force gauge dynamometer (White Oak Orthodontic Products, Pennsylvania, USA) was used to measure the force generated due to wire recoil on unloading.

In Group II, the test premolars were not bonded until a 0.16" x 0.22" NiTi archwire (Ortho Technology, Inc., Florida, USA) is passively engaged in all bracket slots. At this time, the bracket of the test premolar was bonded, the archwire was activated and the produced force was measured exactly as in Group I but the generated intrusive force was much higher in Group II.

In both Group I and Group II, the test premolars were a traumatically extracted after 3 weeks of intrusive force application. ${ }^{9,16}$

\section{Preparation of the specimens for transmission electron microscopy (TEM):- \\ Preparation and fixation: -}

Immediately after carful extraction of each tooth, a sharp horizontal split was done using chisel and hammer into coronal and radicular parts. These parts were instantly immersed in a mixture of $2.5 \%$ glutaraldehyde and $10 \%$ formaldehyde ( $\mathrm{F} / \mathrm{G}$ solution) in a labeled jar for each group. After 5 minutes, the coronal and radicular tooth parts were further split vertically. The coronal and buccal radicular pulpal tissues (subgroups a and b respectively) were collected re-immersed in the fixative solution for 24 hours. After that, the extirpated pulpal specimens were prepared according to (Bancroft and Stevens, 2008) for electron microscopic examination. From each part, cross sections (1 $\mathrm{mm}$ thick) were cut and washed several times in phosphate buffer solution with $\mathrm{pH}$ 7.2-7.4. The specimens were post-fixed in $1 \%$ osmium tetroxide for one hour, and washed again in phosphate buffer. The specimens were loaded in ascending concentrations of ethyl alcohol for complete dehydration. The specimens were embedded in (EPON 812) in flat rubber moulds to obtain the specimen blocks. Semi-thin sections were cut with a diamond knife, mounted on glass slides and stained with $1.0 \%$ toluidine blue for light microscopic examination. The area of interest, peripheral odontoblastic zone, was selected for ultra-thin sectioning. The cut sections were stained with uranyl acetate and lead citrate to be examined with transmission electron microscope (Joel Ltd., Tokyo, Japan). Then, the specimens were thoroughly examined and reported.

\section{Results:-}

The applied force:-

The applied force due to archwire unloading in the light intrusive force group (Group I) ranged from 18 to 35 with a mean of $26.25 \pm 5.18$ grams. In group II where heavier intrusive force was applied, the force was much higher and ranged from 86 to 122 grams with a mean of $104.5 \pm 12.34$ grams (Table 1). 
Table 1:- Descriptive statistics of the applied force (in grams) in Group I and Group II

\begin{tabular}{|l|l|l|l|l|l|l|l|l|l|}
\hline & $\mathrm{n}$ & Mean & SD & SE & Median & Mode & Range & Min. & Max. \\
\hline Group I & 8 & 26.25 & 5.18 & 1.83 & 25.5 & 25 & 17 & 18 & 35 \\
\hline Group II & 8 & 104.5 & 12.34 & 4.38 & 101.5 & --- & 36 & 86 & 122 \\
\hline
\end{tabular}

n: Number of cases $\quad$ SD: Standard deviation $\quad$ SE: Standard error

\section{Control group:-}

Transmission electron microscopic micrographs revealed common cellular features regarding coronal and radicular odontoblasts. They appeared columnar in shape with well-defined cell membrane. Odontoblastic nuclei are oval and most of them were basally situated. The nuclei showed normal chromatin distribution and dominance of euchromatin. There was distinct boundary between condensed and loose chromatin. Their nuclei appeared normal and surrounded by clear regular double nuclear membrane. Cytoplasmic organelles were clearly identified. Well developed strands of rough endoplasmic reticulum (RER) were arranged around the nuclei in the cytoplasm of odontoblasts. The electron-dense strands of RER are parallel to the long axis of the cell in the areas further from the nucleus (the supranuclear zone). Abundant mitochondria were detected in the odontoblastic cytoplasm especially in the apical region of the cells. Although they had a variety of shapes, each of them preserved its typical structure, which is a double membrane with internal cristae. Numerous junctions were seen subjacent to odontoblasts including tight junctions, desmosomes and gap junctions (Figures1 and 2).

\section{Subgroup Ia:-}

Coronal pulpal odontoblasts attained some alterations with wide extracellular compartments. The cell membranes remained intact but ill-defined in most of the samples. Cell junctions between cell membranes were almost lost and wide extracellular compartments were markedly observed. Odontoblastic nuclei attained their normal regular double nuclear membrane with slight peripheral or central chromatin clumping in most samples. The cytoplasm was dark homogenous occupied with cytoplasmic organelles. Rough endoplasmic reticulum were numerous and apically situated in many specimens. Multiple mitochondria were detected in some of the specimens but they were swollen and lost their internal cristae (Figures 3-6).

\section{Subgroup Ib:-}

Electron micrograph of radicular pulpal tissue showed diversity of odontoblastic cellular and nuclear patterns. Cells showed wide range of different sizes. All possessed wide extracellular matrixes and complete loss of cellular junctions. Irregular cell membranes were a common finding in all specimens. Six specimens expressed single nucleus but with prominent chromatin condensation. Two other specimens showed multiple small pychnotic nuclei. Various stages of nuclear division were also detected. The cytoplasmic-nuclear ratio was variable among the specimens. The cytoplasm was dark homogenous with ill-defined organelles. Rough endoplasmic reticulum was illdefined in most specimens. Multiple faint swollen mitochondria were detected in some of the specimens. Few small sized cytoplasmic electron-dense vesicles were detected in two specimens of this subgroup (Figures 7-10).

\section{Subgroup IIa:-}

Coronal peripheral pulpal cells showed few regular odontoblastic cells with regular configuration regarding their cell membranes and nuclei. Other cells showed regular outlines but associated with diffuse nuclear chromatin condensation and numerous para-nuclear electron-dense vesicles. Many odontoblastic cells were multinucleated and loaded with numerous intracytoplasmic electron-dense vesicles (Figures 11-14).

\section{Subgroup IIb:-}

Radicular odontoblastic cells expressed nearly the same ultrastructural pattern. All of the cells appeared shrunken with irregular cell membranes. Cellular junctions were ill-defined with moderate extracellular matrix. The common characteristic feature of all cellular element of this subgroup was multinucleation. Their cytoplasm was dark homogenous with ill-defined cytoplasmic organelles. Electron-dense intracytoplasmic vesicles vanished out in this subgroup. Monocytes obviously detected in the field in some specimens of this subgroup. It was characterized by electron-lucent cytoplasm with a moderately developed rough endoplasmic reticulum (figures15-18).

\section{Discussion:-}

Successful orthodontic treatment depends on moving the teeth as efficiently as possible with no or least damage to teeth and their supporting tissues. This study investigated the effect of two magnitudes of intrusive orthodontic force 
on pulpal odontoblasts in the human premolars after an interval of three weeks. The variables studied were two magnitudes of intrusive force (independent variable) and their effect on selected area of pulpal tissues, namely the odontoblasts which may accordingly have important clinical relevance.

It was of prime importance to study the effects of the intrusive forces on pulp tissue which is as normal and healthy as possible like in normal clinical conditions. Therefore, the inclusion criteria were set very carefully to select healthy young pupal tissue which is not affected by any disease, trauma or medicine as possible. Ethical considerations aimed to minimize patients' discomfort and damage to their teeth. So patients were allowed to take a pain killer with no anti-inflammatory effect (Paracetamol).

Regarding clinical application of intrusive force, it was set to resemble the force applied during leveling stage with 0.012 " archwires and during finishing and detailing stage with the $0.016 " \mathrm{x} 0.022$ " archwires. Hence, the brackets of the test premolars were bonded only $1 \mathrm{~mm}$ occlusal to the archwire level which is a common malposition during both treatment stages. Although this mechanics may not apply pure intrusion and will be accompanied by little anticlockwise labiopalatal moment, this momental component seems to be much less than that expected during intrusion applied with cantilever arm from first molar to first premolar (Patel et al., 2010). The mean of applied light force (26.25 grams) was not far from the mean optimum intrusive force reported by (Proffit et al.,2012) for multirooted teeth (20 grams), while the mean of the applied heavy force was much higher (104.5 grams).

The used bracket system was Synergy Low-Friction Straight Wire (LFSW) appliances with 0.022" slot (Rocky Mountain Orthodontics RMO Corp., Colorado, USA). Both the 0.022" slot and its design in Synergy brackets allow more archwire play especially at the mesial and distal ends of the slot. This makes the actually applied force very close to that measured by the dynamometer.

Extraction was done 3 weeks after intrusive force application. This period was reported by other workers ( Ramazanzadeh et al., 2009) and (Lazzaretti et al., 2014) to be sufficient for the intrapulpal chronic inflammatory process to take place.

Extirpation of pulp tissue was selected, in order to minimize the effect of any external factors, such as decalcification, except the applied force. Immediate tooth splitting and immersion in the fixative after extraction was done, that ensured profound fixation and preservation of the tissues before any manipulation or dissection of pulp from dentin. Peripheral pulp tissues were dissected carefully to ensure presence of odontoblastic cell layer. Ultrastructural cellular details were optimally obtained by transmission electron microscope.

Evidence of affection of the odontoblasts could be manifested in all experimental groups as they expressed various degrees of nuclear alterations, chromatin condensation, cytoplasmic organelles affection and loss of cellular junctions. The degenerative changes and aspiration of odontoblasts, were reported by other researchers due to intrusive force application for 21 days (Stenvick et al., 1970) ,Mostafa et al., 1991), (Shigehara et al., 2006) and (Ramazanzadeh et al., 2009). Cells are constantly adjusting to the mechanical influences of their surroundings, starting a complex mechano-chemical feedback, which depends on mechano-transduction mechanisms. Whereas adhesion structures have been shown to play a central role in mechano-transduction, it now emerges that the nucleus may act as a mechano-sensitive structure (Nejma et al., 2016).

In all experimental groups, loss of odontoblastic orientation is noticed and evident by loss of cellular junctions and wide extracellular compartments. This could be attributed to the sensitivity of odontoblasts, and they are easily disturbed by changes in their external environment such as intrusive force. Application of orthodontic force, induce mechanical strain which could stimulate the cells and their associated extracellular matrix. This stimulation has the ability to regulate integrin expression, focal adhesion proteins, cytoskeletal organization, cell morphology, cell adhesion to extracellular matrices, cell proliferation, and cell differentiation (Masella and Meister ,2006).

When the extracellular matrix is stressed, isometric tension develops in the cells within the matrix. This isometric tension is equal in magnitude to the mechanical tensional force exerted upon them by the extracellular matrix, leading to changes in their cellular cytoskeleton and architecture with activation of cellular transcription factors( Masella and Meister ,2006) and (Cukierman et al., 2002). 
This in turn influences the expression of genes involved in cell attachment, proliferation, differentiation, and apoptosis.

Loss of odontoblastic cells junctions of both coronal and radicular parts in all experimental groups indicated that both light and heavy intrusive orthodontic forces affect cellular junctions. This may go with (Gajapuradal et al., 2016) who reported that even small forces of short duration of around 4 hours may be adequate to evoke cellular responses.

Odontoblastic nuclear alteration was very interesting finding in this study. Under light intrusive force, coronal odontoblasts expressed normal nuclear membrane and signs of chromatin condensation. Although, most radicular odontoblasts expressed single nucleus but with prominent chromatin condensation, few cells showed multiple small pychnotic nuclei and various stages of nuclear division were detected. Also, cytoplasmic electron dense vesicles of different sizes detected in radicular cells.

Under heavy intrusive force, many coronal odontoblastic cells were multinucleated and loaded with some intracytoplasmic perinuclear electron-dense vesicles. On the other hand, most radicular odontoblasts were multinucleated with disappearance of perinuclear electron-dense vesicles.

Apparently, odontoblastic nuclei were mostly affected and nuclear division was seen in many electron micrographs of experimental groups, which suggests that the mechanical forces transmitted through the cell could result in internal remodeling of the nuclear architecture and chromatin arrangement. The results revealed that the effect of heavy intrusive force was greater than light force on odontoblastic cells. Furthermore, the impact of the transmitted force was greater on radicular rather than coronal cells that were demonstrated by complete nuclear division and odontoblastic differentiation into odontoclastic cells particularly in radicular portions. Apparently, odontoblasts transform and differentiate into multinucleated cells, which could be odontoclasts, when subjected to mechanical intrusive force.

Intracytoplasmic paranuclear electron-dense granules that were detected in some odontoblasts may indicate cellular activity prior to nuclear division. This explains why these granules were very few and small in subgroup Ib (few nuclear divisions), abundant in subgroup IIa (more nuclear divisions) and almost entirely absent in subgroups Ia and IIb (no nuclear division and completed nuclear division respectively). More investigations are required regarding that point.

Multinucleation of odontoblastic cells brings us about odontoclastic transformation and subsequent internal root resorption under the influence of intrusive force. Differentiation of odontoclasts in coronal pulpal highlight the idea of internal resorption could be elicited coronal in response to heavy intrusive force. This is in agreement with (Weiland, 2006) who concluded that the type and magnitude of orthodontic forces play a major role in root resorption. He also stated that continuous forces, as produced by materials with pseudo-elastic characteristics, seem to produce more resorption than discontinuous forces and the magnitude of the orthodontic force up to $200 \mathrm{cN}$ is probably not decisive for root resorption.

Blood monocytes have been detected in close approximation to radicular differentiated odontoclasts at the site of heavy force. Furthermore, mononuclear cells were found adjacent to degenerating odontoclasts and resorbed enamel surface from which the odontoclasts had moved or detached in the transitional stage of shedding (Sahara et al., 2004). This might indicate completion of resorptive process in the radicular part under heavy forces.

Cytoplasmic organelles disruption was noticed throughout the experimental groups such as swollen mitochondria. This suggests that even under minimal intrusive force affection of cytoplasmic organelles occur.

We carried out this study in an attempt to discover whether, how and when orthodontic intrusion could be a cause for internal tooth resorption. 


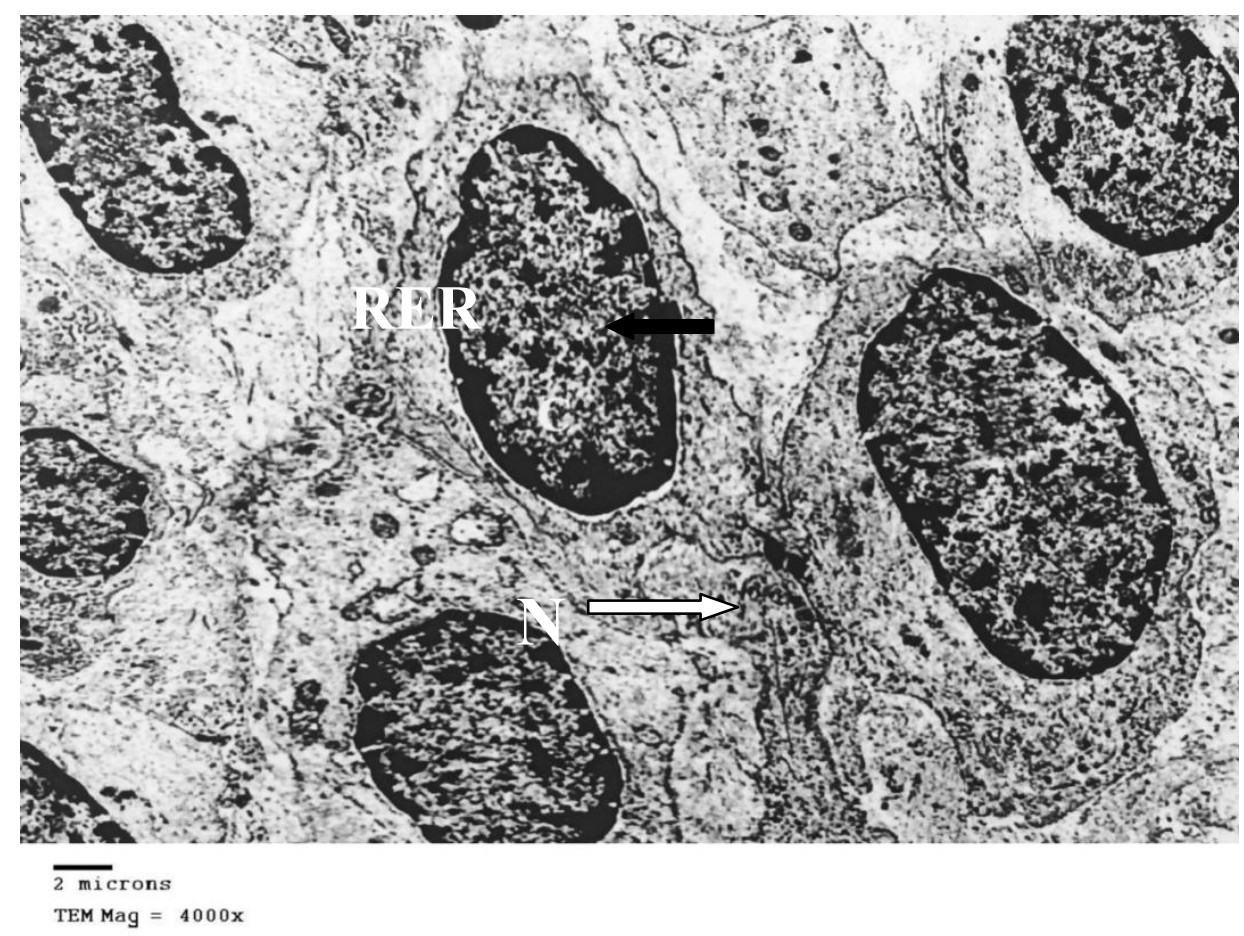

Fig. 1:- Electron micrograph showing normal coronal odontoblasts with palisading pattern, normal cell junctions (white arrow) and nuclear membranes (black arrow).

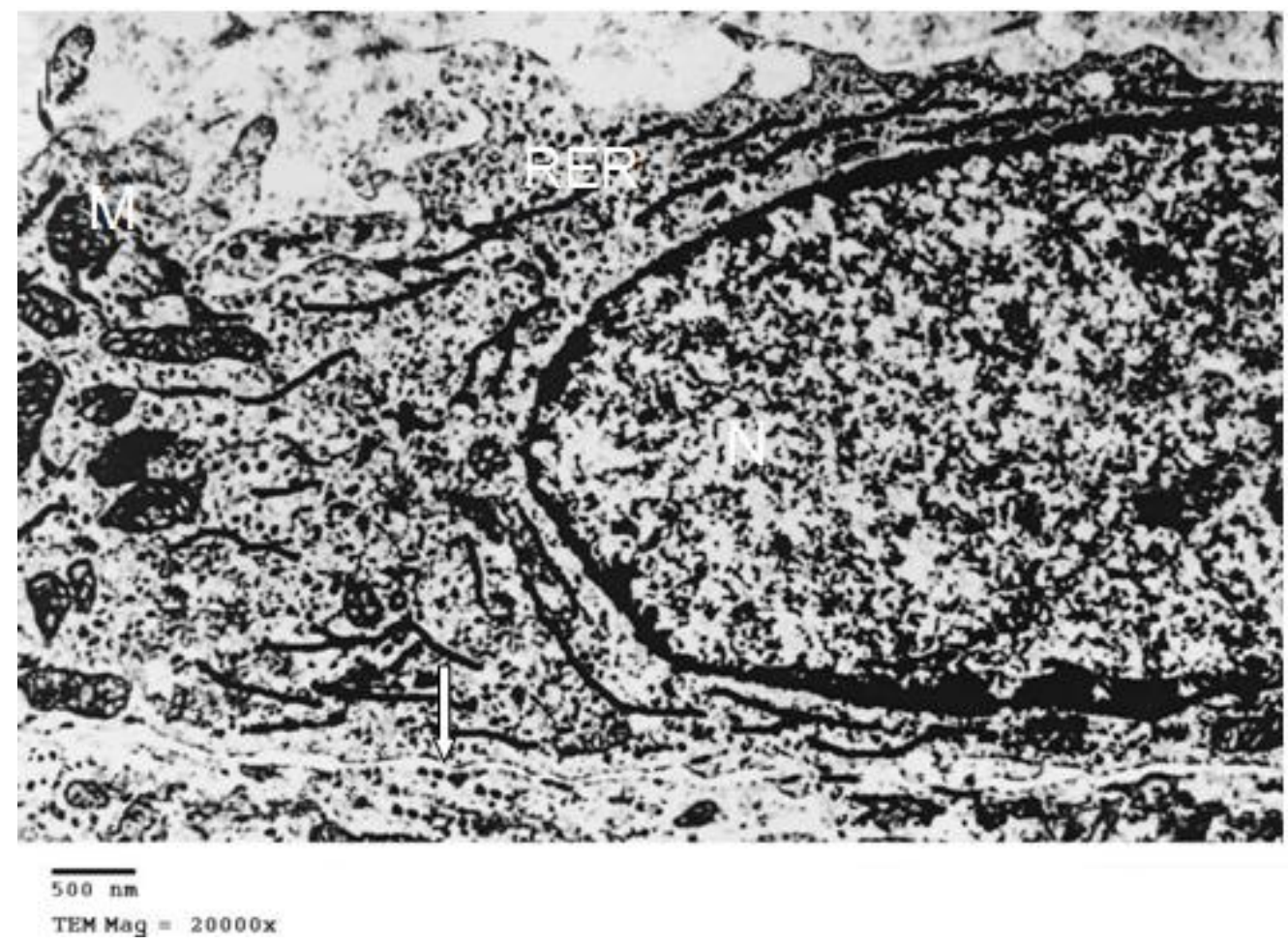

Fig. 2:- Electron micrograph showing normal odontoblasts with normal nuclei, chromatin distribution, cytoplasmic organelles ;nucleas (N) mitochondria (M ), rough endoplasmic reticulium (RER) and cell junction (arrow). 


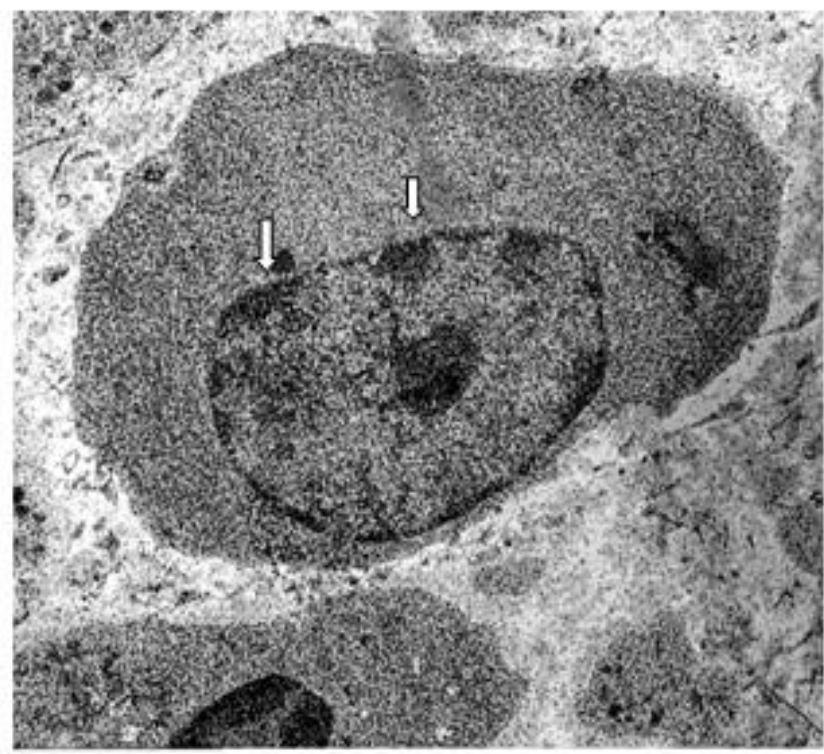

incrases

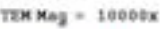

Fig.3 Electron micrograph of pulpl tissue subgroup Ia showing odontoblasts with regular cell membrane, dark homogenous cytoplasm, regular nuclear membrane with peripheral chromatin condensation (arrows).

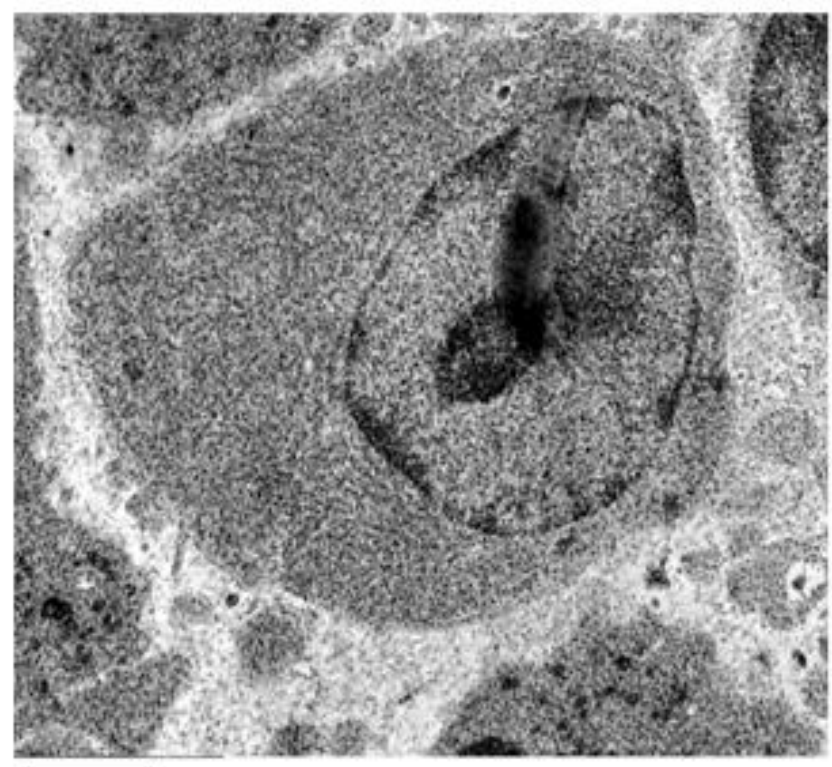
$\overline{2 \text { nicrons }}$

Fig.5 Electron micrograph of subgroup Ia showing odontoblasts with ill-defined cellular junctions, wide extracellular matrix and numerous rough endoplasmic reticulum. Nuclear chromatin condensation is slightly detected peripherally.

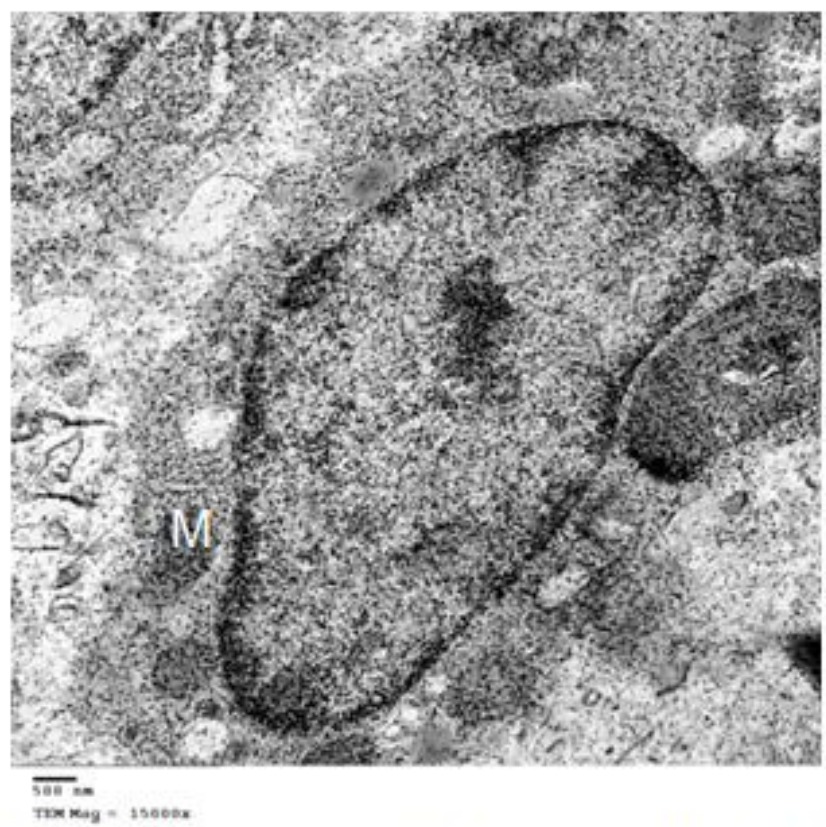

Fig. 4 Electron micrograph of subgroup Ia showing odontoblasts with ill defined cell membrane, homogenous cytoplasm, swollen mitochondria $(M)$, open faced nucleus with regular nuclear membrane, slight peripheral chromatim

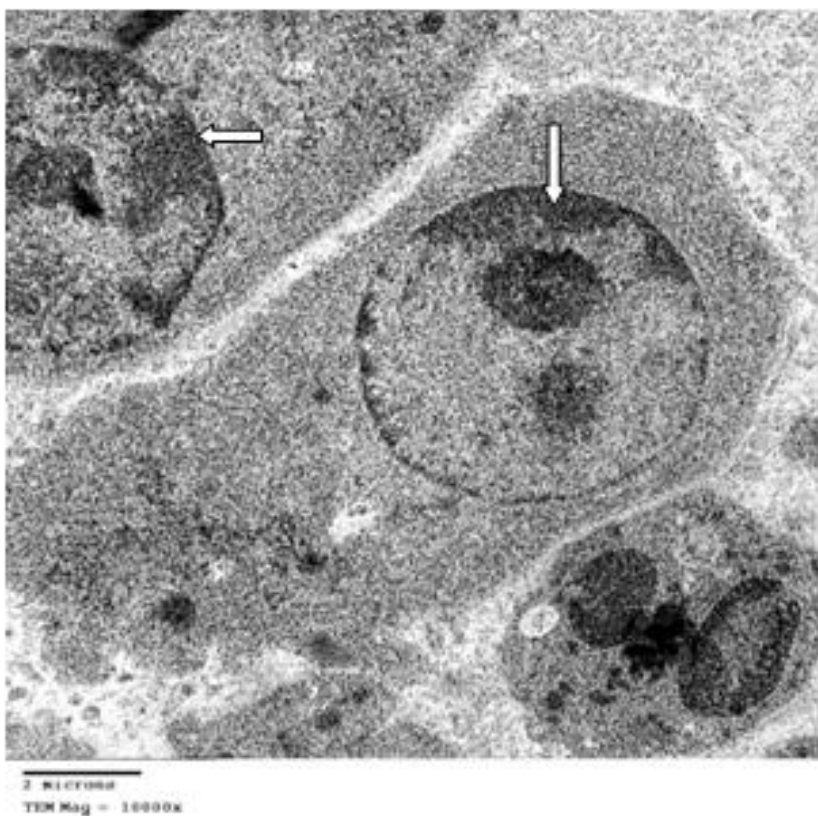

Fig.6 Electron micrograph of subgroup Ia showing signs of peripheral nuclear chromatin condensation (arrows). 


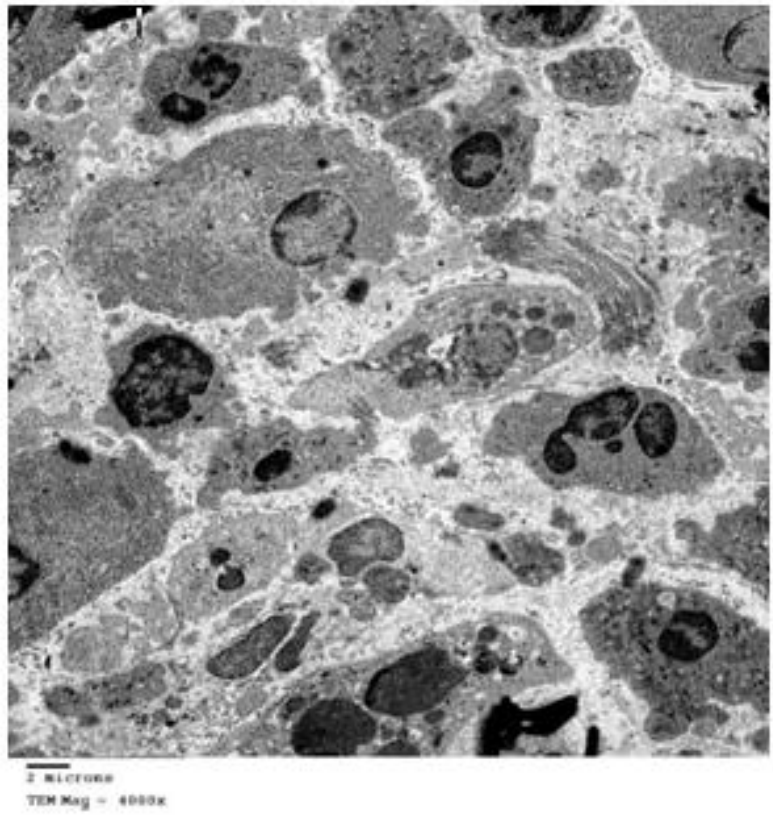

Fig.7 Electron micrograph of radiculal pulpal tissue (subgroup Ib) showing diverse odontoblastic pattern, very wide extracellular compartments and various stages of nuclear division.

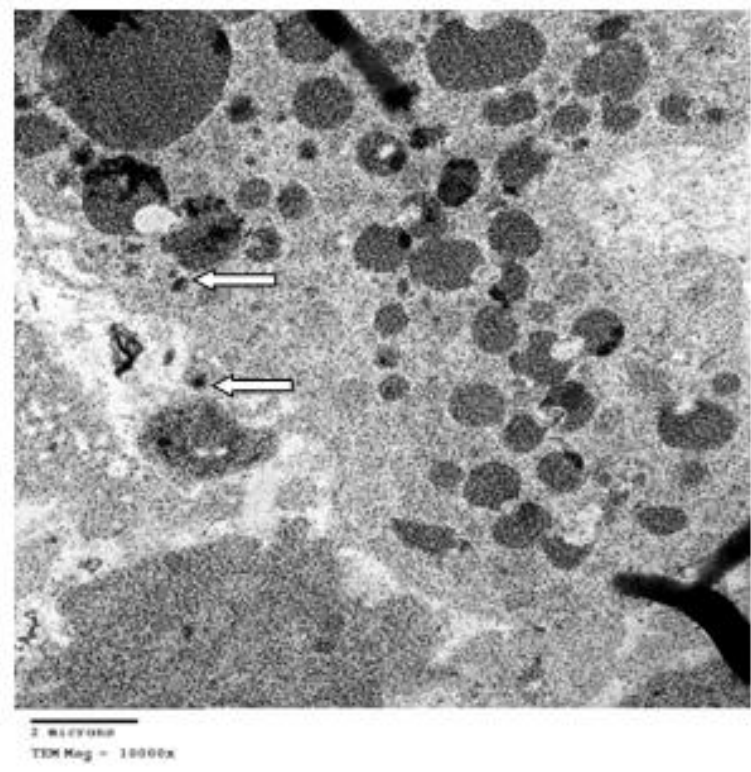

Fig.9 Electron micrograph of subgroup Ib showing electrondense cytoplasmic vesicles of various sizes occupying odontoblastic cytoplasm (arrows).

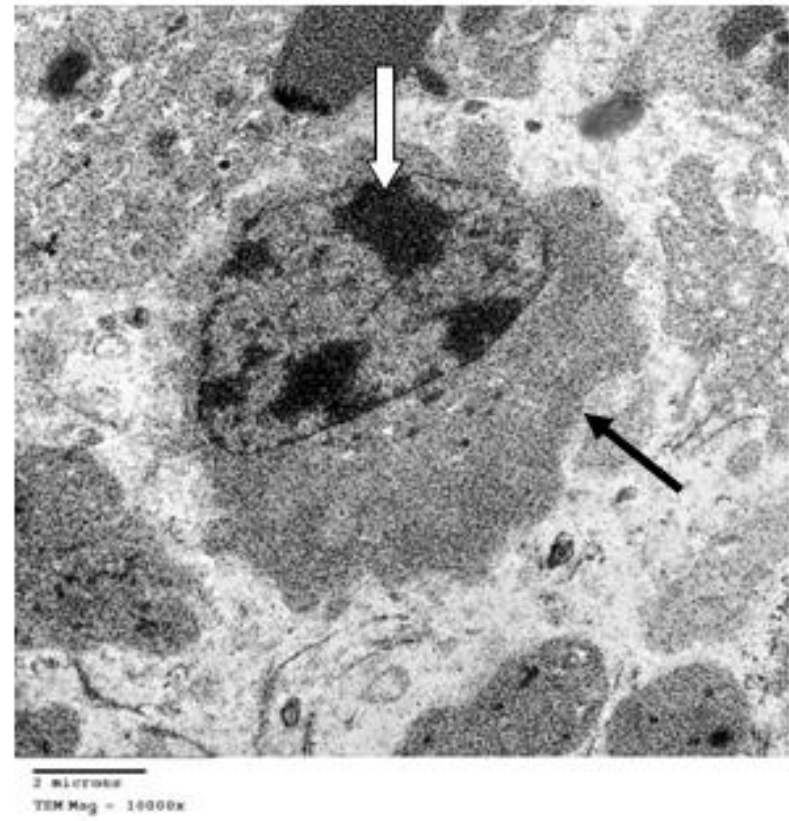

Fig.8 Electron micrograph of subgroup Ib showing odontoblasts with ill-regular cell membrane (black arrow), shrunken homogenous cytoplasm, large nucleus with prominent diffuse and peripheral chromatin condensation (white arrow).

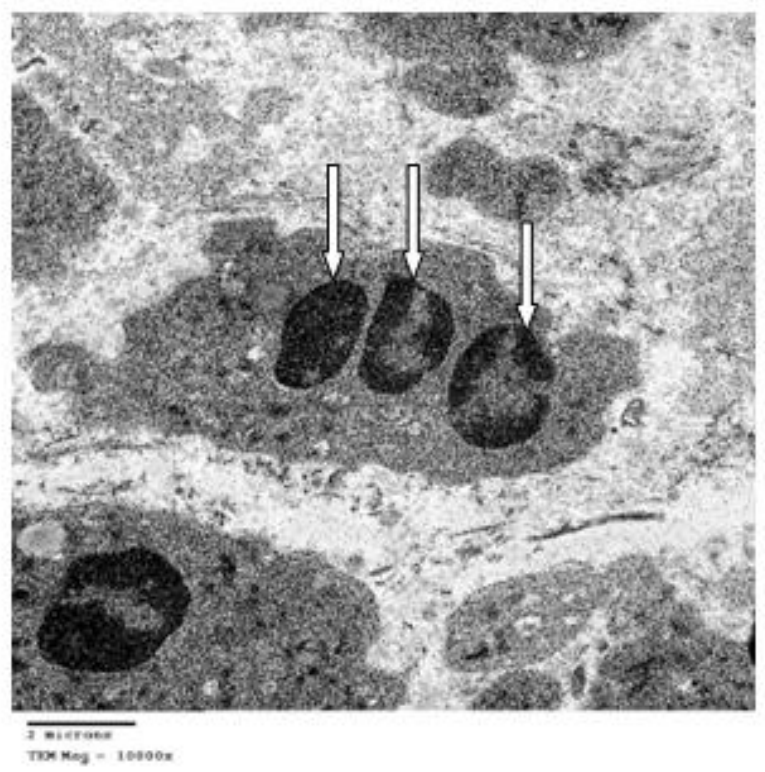

Fig.10 Electron micrograph of subgroup Ib showing an odontoblast with multiple small pychnotic nuclei. 


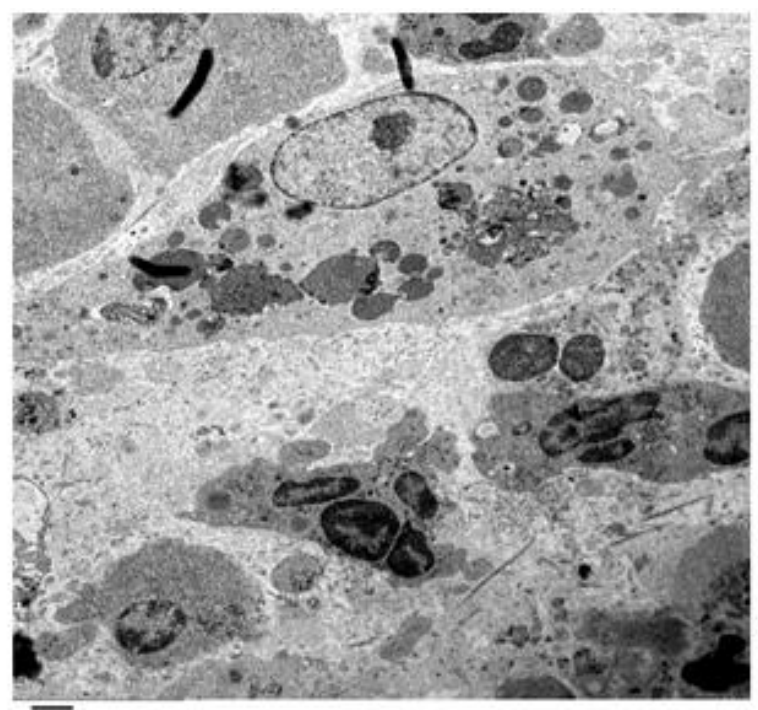
$\overline{\text { Tieress }}$

Fig.11 Electron micrograph of subgroup $\mathrm{Ila}$ showing both regular odontoblasts and multimucleated smaller irregular cells.

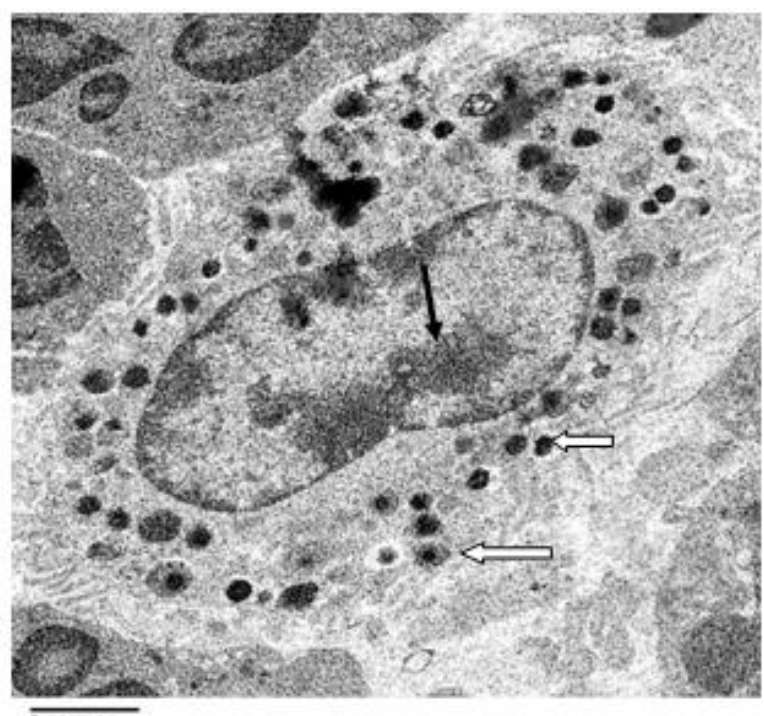

$\overline{T \text { mierana }}$

Fig.12 Electron micrograph of subgroup $\mathrm{\Pi l}$ showing an odontoblast with ill-defined cell membrane, intracytoplasmic perinuclear electron-dense vesicles (arrows) and regular nuclear membrane with diffuse chromatin condensation (black arrow).

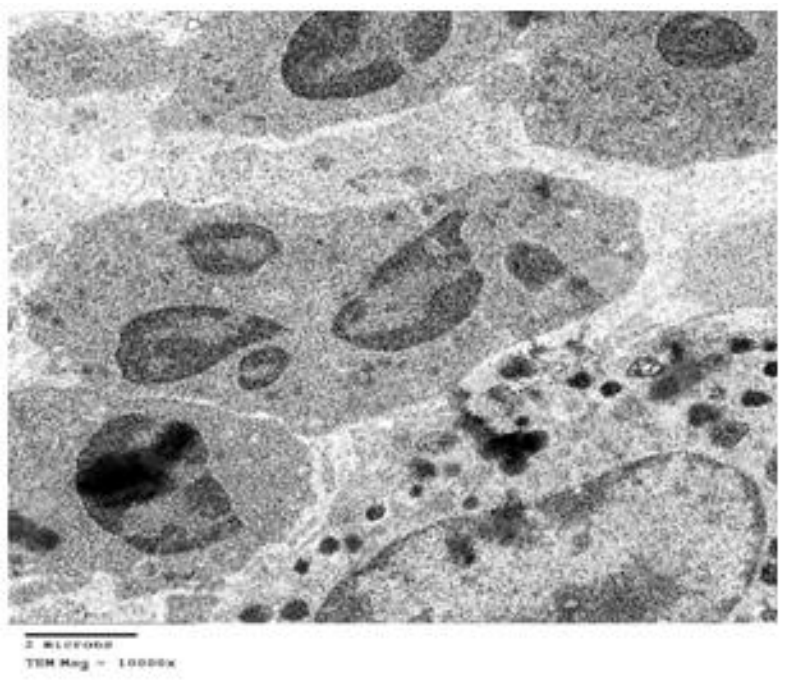

Fig.13 Electron micrograph of subgroup Ila showing a multinucleated odontoblast.

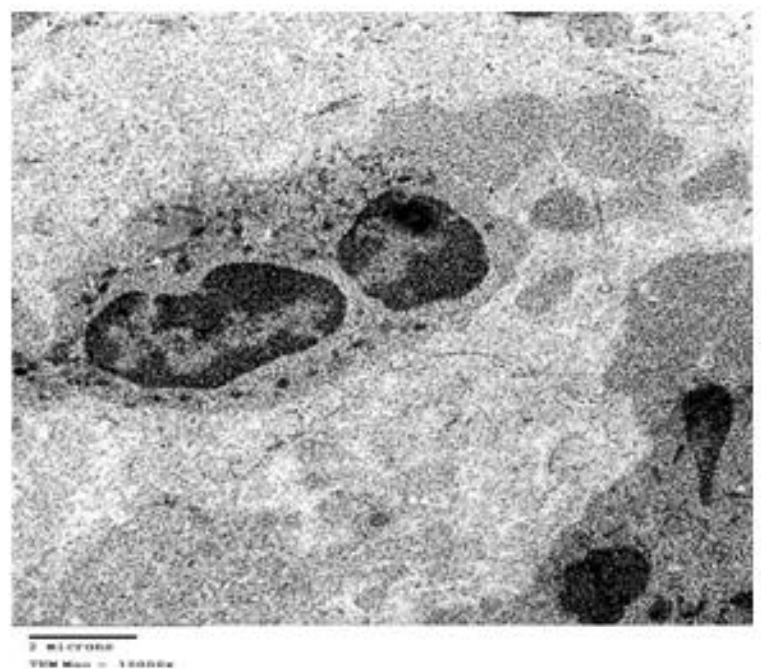

Fig.14 Electron micrograph of subgroup Ila showing a multinucleated odontoblast with evidence of intracytoplasmic electron-dense vesicles. 


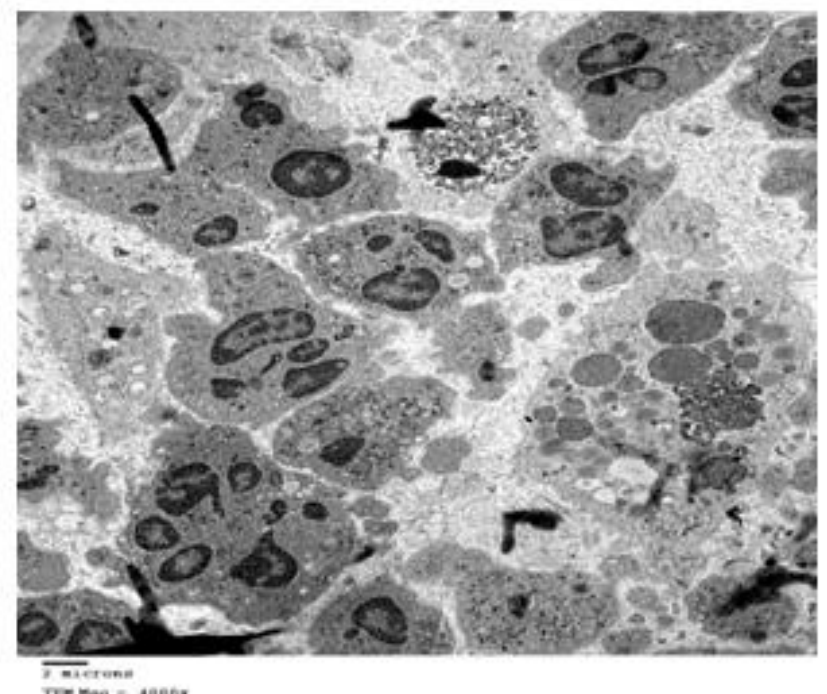

Fig.15 Electron micrograph of radicular pulp tissue of subgroup Ilb showing condensation of organized multimucleated cells.

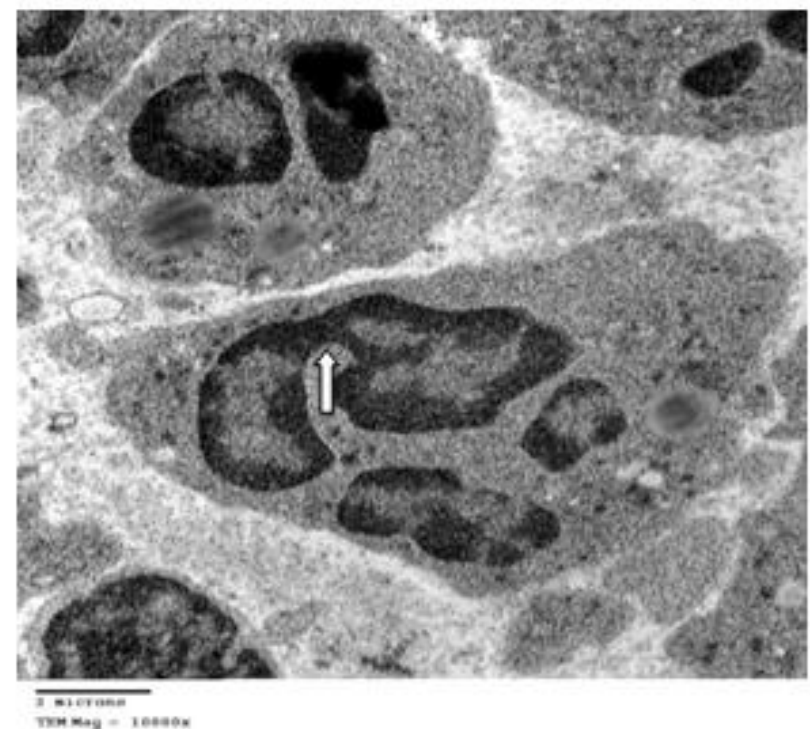

Fig.17 Electron micrograph of subgroup IIb showing nuclear constriction (arrow).

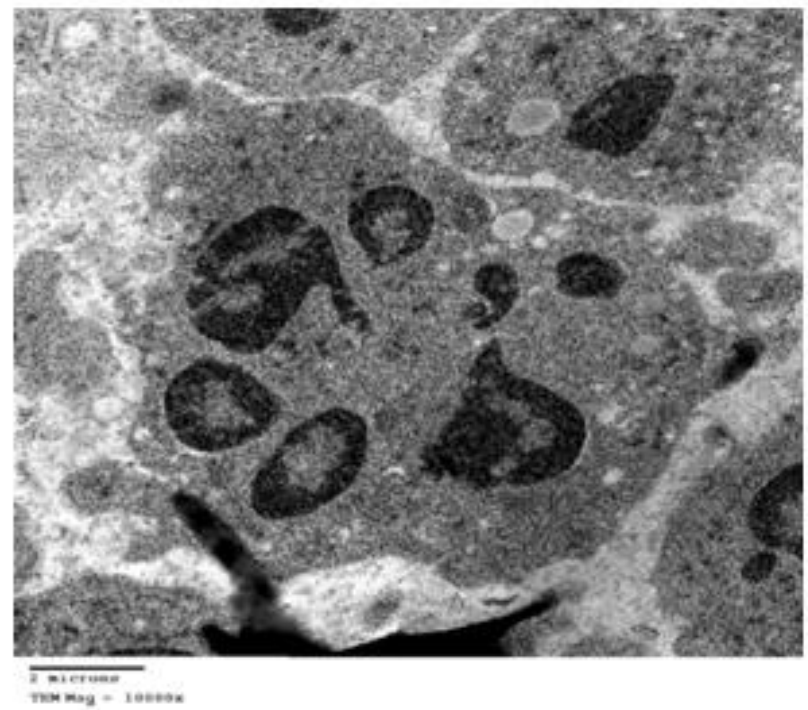

Fig.16 Electron micrograph of subgroup $\mathrm{Ilb}$ showing a multinucleated cell with irregular nuclear membrane and vacuolated cytoplasm.

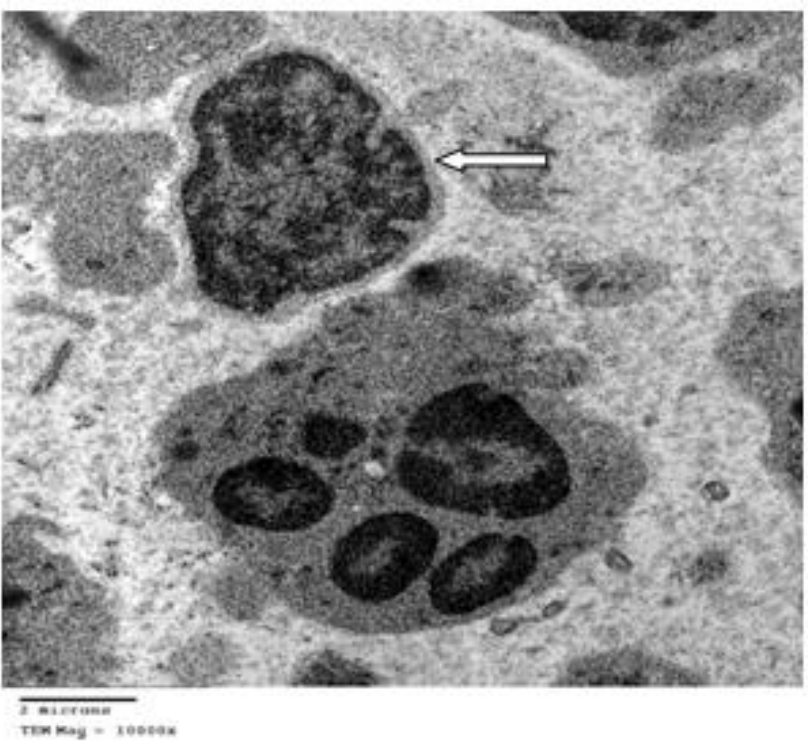

Fig.18 Electron micrograph of subgroup IIb showing a multimucleated cell in close association with monocyte (arrow).

\section{Conclusions:-}

Intrusive orthodontic force for three weeks interval can evoke serious ultra-structural changes in odontoblastic cells. Heavy intrusive have greater impact on odontoblastic cells than light force. Generally, radicular cells are more affected than coronal cells by the same intrusive force magnitude. Both coronal and radicular odontoblastic cells can transform into multinucleated cells under the influence of heavy intrusive orthodontic force. Even light intrusive force could induce few multi-nucleation of radicular odontoblastic cells. 


\section{Recommendations:-}

1. Further investigations are needed using different techniques such as immunohistochemical and decalcified tooth sections to confirm the phenotype of the multinucleated cells and their effect on dentin.

2. Further studies are recommended using different periods of force application to investigate whether the force impact is transient or permanent on odontoblastic cells which might help in case prognosis.

3. Orthodontic intrusion must be performed with ultimate caution and full awareness of the applied force.

\section{References:-}

1. Andreasen JO, Andreasen FM, Andreasen L. Textbook and color atlas of traumatic injuries to the teeth. 4th ed. 2007; Oxford.

2. Bancroft JD, Stevens DB. Theory and practice of histology techniques. $2^{\text {nd }}$ ed. The CV Mosby Company, St Louis, 2008.

3. Cukierman E, Pankov R, Yamada KM. Cell interactions with three-dimensional matrices. Curr Opin Cell Biol. $2002 ; 14: 633-639$.

4. Gajapuradal J, Deshmukh C, Biradar A, Podar S, Bhalkeshwar, Bansal A, Zubair W. Pulpal response to orthodontic treatment: A review. IOSR-J Dent Med Sci. 2016; 15:73-75.

5. Hamilton RS, Gutmann JL. Endodontic-orthodontic relationships: a review of integrated treatment planning challenges. Int Endod J. 1999; 32:343-360.

6. Harris DA, Jones AS, Darendeliler MA. Physical properties of root cementum, Part 8. Volumetric analysis of root resorption craters after application of controlled intrusive light and heavy orthodontic forces: A microcomputed tomography scan study. Am J Orthod Dentofac Orthop. 2006; 130:639-647.

7. Krishnan V, Davidovitch Z. Cellular, molecular, and tissue-level reactions to orthodontic force. Am J Orthod Dentofac Orthop. 2006; 129:469-501.

8. Lazzaretti DN, Bortoluzzi GS, Fernandes LFT, Rodriguez R, Grehs RA, and Hartmann MSM. Histologic evaluation of human pulp tissue after orthodontic intrusion . J Endod. 2014; 40:1537-1540.

9. Masella RS, Meister M. Current concepts in the biology of orthodontic tooth movement. Am J Orthod Dentofac Orthop. 2006; 129:458-468.

10. Mostafa YA, Iskander KG, El-Mangoury NH: Iatrogenic pulpal reactions to orthodontic extrusion. Am J Orthod Dentofac Orthop. 1991; 99:30-34.

11. Nejma B, Julien A and Christophe G: Under pressure; mechanical stress management in the nucleus. Cells, 2016; 5:27-38.

12. Orban BJ, Bhaskar SN. Orban's Oral Histology and Embryology. $18^{\text {th }}$ ed., The CV Mosby Company, St Louis, 1976.

13. Patel S, Ricucci D, Durak C, Tay F. Internal root resorption: A review. J Endod. 2010; 36:1107-1121.

14. Proffit W, Fields H, Sarver D. Contemporary Orthodontics. 5th Ed, Mosby, St Louis, 2012.

15. Ramazanzadeh BA, Sahhafian AA, Mohtasham N, Hassanzadeh N, Jahanbin A, Shakeri MT. Histological changes in human dental pulp following application of intrusive and extrusive orthodontic forces . J Oral Sci. 2009; 51: 109-115.

16. Sahara N, Ozawa H. Cementum-like tissue deposition on the resorbed enamel surface of human deciduous teeth prior to shedding. Anat Rec. 2004; 279A:779-791.

17. Shigehara S, Matsuzaka K, Inoue T. Morphohistological change and expression of HSP70, osteopontin and osteocalcin mRNAs in rat dental pulp cells with orthodontic tooth movement. Bull Tokyo Dent Coll. 2006; 47:117-124.

18. Stenvick A, Mjör IA. Pulp and dentine reactions to experimental tooth intrusion. A histologic study of the initial changes. Am J Orthod. 1970; 57:370-385.

19. Veberiene R, Smailiene D, Danielyte J, Toleikis A, Dagys A, Machiulskiene V. Effects of intrusive force on selected determinants of pulp vitality. Angle Orthod. 2009;79:1114-1118.

20. Villa PA, Oberti G, Moncada CA, Vasseur O, Jaramillo A, Tobòn D, Agudelo JA. Pulp-Dentine Complex Changes and Root Resorption During Intrusive Orthodontic Tooth Movement in Patients Prescribed Nabumetone. J Endod. 2005; 31:61-66.

21. Weiland F: External root resorptions and orthodontic forces: correlations and clinical consequences. Prog Orthod. 2006; 7:156-163.

22. Yamaguchi M, Kasai K. The effects of orthodontic mechanics on the dental pulp. Semin Orthod, 2007; 13:272280. 